Active and Passive Elec. Comp., June 2004, Vol. 27, pp. 119-123

\title{
A NEW PARAMETER-EXTRACTION METHOD FOR DGS AND ITS APPLICATION TO THE LOW-PASS FILTER
}

\author{
HAIWEN LIU ${ }^{\mathrm{a}, \mathrm{b}, *}$, XIAOWEI SUN ${ }^{\mathrm{b}}$ and ZHENGFAN LI ${ }^{\mathrm{a}}$ \\ ${ }^{a}$ Department of Electronic Engineering, Shanghai Jiao Tong University, P.O. Box 006, No. 1954, \\ Huashan Road, Shanghai 200030, China; ${ }^{\mathrm{b}}$ Shanghai Institute of Microsystem and Information \\ Technology, Chinese Academy of Sciences, Shanghai 200050, China
}

(Received 8 April 2003; In final form 28 July 2003)

\begin{abstract}
A new and simple parameter-extraction method for the equivalent circuit of defected ground structure (DGS) is presented. Using this method, circuit simulation, based on the DGS equivalent-circuit model, show excellent agreements with the electromagnetic (EM) simulation. Further, our method is applied effectively to design a low-pass filter (LPF) with DGS. Comparison between simulation and measurement confirm the validity of the LPF configuration and design procedure. Simple structure and high power handling capability are obtained from the proposed LPF.
\end{abstract}

Keywords: Defected ground structure; Parameter-extraction; Low-pass filter

\section{INTRODUCTION}

Defected ground structure (DGS) for the microstrip line, which has period etched defects in the backside metallic ground plane, is one recent hotspot of microwave circuit design [1-3]. Compared to photonic bandgap (PBG) [4-6], DGS has simple structure and potentially great applicability to design microwave circuits such as couplers, amplifiers and oscillators [7-9].

Generally, DGS is composed of two square defected areas and a connecting slot, and can provide the bandgap characteristics in certain frequency bands. The bandgap characteristics can be explained by an equivalent circuit of DGS using a parallel $\mathrm{L}-\mathrm{C}$ resonator.

In this paper, radiating effects are taken into account. The proposed equivalent circuit includes a parallel resistor to explain the radiating effects, and a new parameter-extraction method for the equivalent circuit is developed. To show the validity of the method, an LPF having two DGS patterns and a T-junction microstrip line is designed and fabricated. The results reveal that the proposed method outperforms the basic method described previously $[1,2]$.

\footnotetext{
* Corresponding author. Tel.: (86-21) 62511070 ext. 8603; Fax: (86-21) 62933001;

E-mail: haiwen_liu@sjtu.edu.cn
} 


\section{DEFECTED GROUND STRUCTURE PATTERN AND PARAMETER-EXTRACTION PROCEDURE}

A microstrip line with a dumb-shaped DGS unit and its equivalent circuit are shown in Figure 1. The equivalent circuit can be represented with a parallel $\mathrm{R}-\mathrm{L}-\mathrm{C}$ resonator. The equivalent circuit parameters, $R_{\mathrm{r}}, L_{\mathrm{r}}$, and $C_{\mathrm{r}}$, can be derived from the S-parameters based on the electromagnetic (EM)-simulation. Once the S-parameters are obtained, these parameters can be extracted by using the relation between the S-parameters and ABCD-parameters as follows:

$$
\begin{aligned}
B & =\left.\frac{\left(1+S_{11}\right) \times\left(1+S_{22}\right)-S_{12} S_{21}}{2 S_{21}}\right|_{\omega=\omega_{\mathrm{o}}}=Z_{\mathrm{r}}, \\
Y_{\mathrm{r}} & =\frac{1}{Z_{\mathrm{r}}} \\
Y_{\mathrm{r}} & =\frac{1}{R_{\mathrm{r}}}+j B_{\mathrm{r}}=\frac{1}{R_{\mathrm{r}}}+j\left(\omega_{\mathrm{o}} C_{\mathrm{r}}+\frac{1}{\omega_{\mathrm{o}} L_{\mathrm{r}}}\right), \\
R_{\mathrm{r}} & =\operatorname{Re}\left(\frac{1}{Y_{\mathrm{r}}}\right), \\
C_{\mathrm{r}} & =\frac{B_{\mathrm{r}}}{\omega_{\mathrm{o}} \cdot\left(\omega_{\mathrm{c}} / \omega_{\mathrm{o}}-\omega_{\mathrm{o}} / \omega_{\mathrm{c}}\right)}, \\
L_{\mathrm{r}} & =\frac{1}{\omega_{\mathrm{o}}^{2} C_{\mathrm{r}}},
\end{aligned}
$$

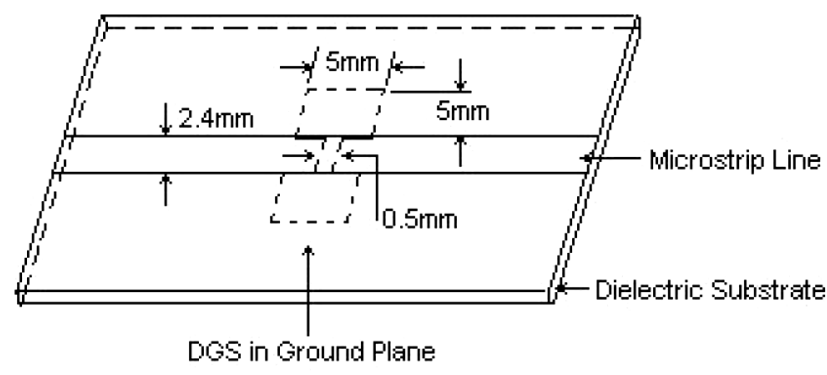

a

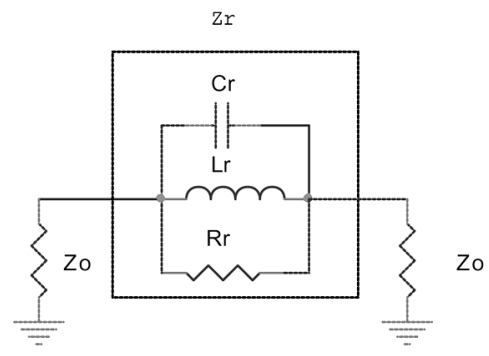

b

FIGURE 1 Configuration of a microstrip line with DGS and its equivalent circuit: (a) configuration of a microstrip line with DGS (dielectric substrate: $\varepsilon_{\mathrm{r}}=2.22$, thickness $=30 \mathrm{mils}$ ); (b) equivalent circuit of a microstrip line with DGS. 


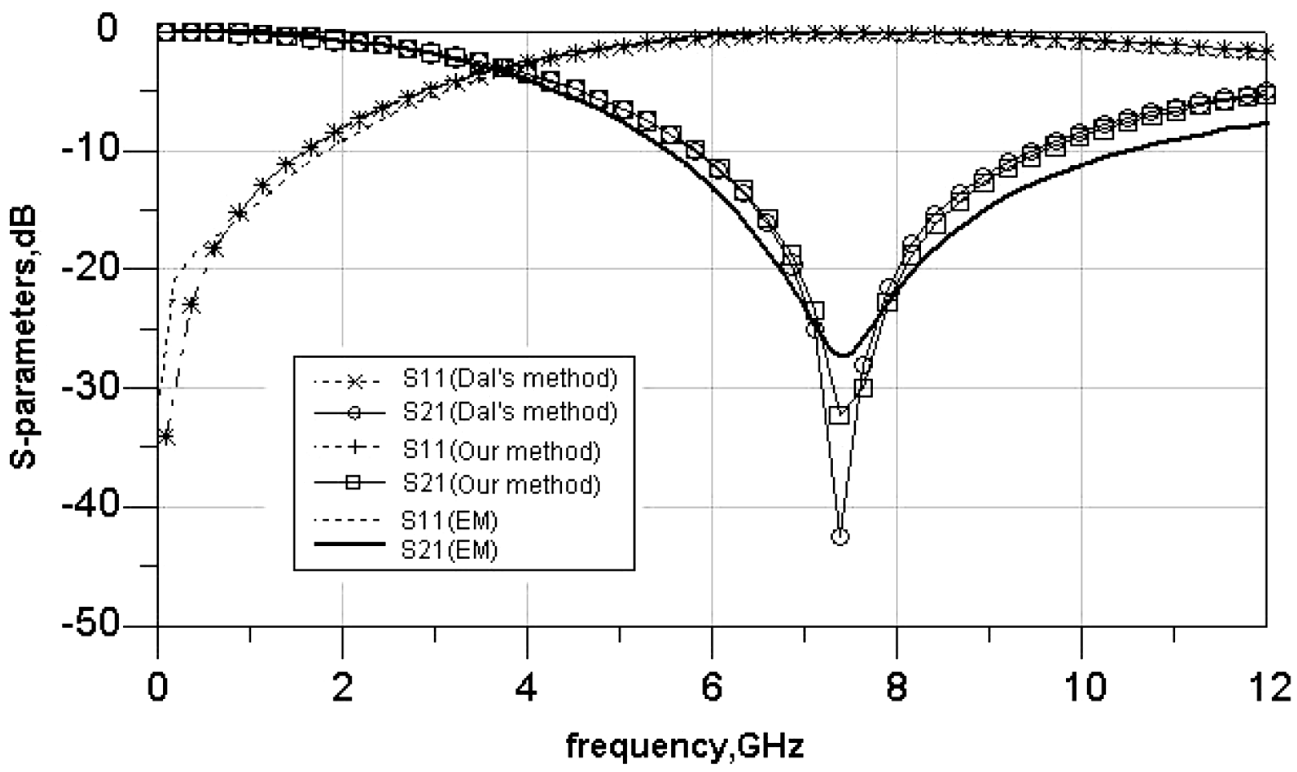

FIGURE 2 Characteristics of a microstrip line with DGS.

where $\omega_{\mathrm{o}}, \omega_{\mathrm{c}}, Z_{\mathrm{r}}, Y_{\mathrm{r}}$, and $B_{\mathrm{r}}$ are the resonant frequency, $3 \mathrm{~dB}$ cutoff frequency, the impedance, the admittance and the conductance, respectively. $\operatorname{Re}(Z)$ denotes the real part of $Z$.

The values of $\omega_{\mathrm{o}}$ and $\omega_{\mathrm{c}}$ are 7.42 and $3.74 \mathrm{GHz}$, respectively, which are obtained from the EM-simulation as shown in Figure 2. Calculated by Eqs. (1)-(5), the extracted values of $C_{\mathrm{r}}, L_{\mathrm{r}}$, and $R_{\mathrm{r}}$ are $0.1426 \mathrm{pF}, 3.1721 \mathrm{nH}, 6121 \mathrm{Ohm}$, respectively, while the extracted values of $C_{\mathrm{r}}$ and $L_{\mathrm{r}}$ are $0.1449 \mathrm{pF}$ and $3.1743 \mathrm{nH}$, respectively, obtained by the basic method described in Ref. [2]. The transmission characteristics of the microstrip line with DGS were simulated using the above-extracted values and are shown in Figure 2, together with EM-simulation. In Figure 2, the circuit simulations show excellent agreements with the EM-simulation. Also, the results reveal that the proposed method outperforms the previous basic method. The circuit simulations are completed by Agilent ADS 2002.

\section{DESIGN OF MICROWAVE LOW-PASS FILTERS WITH DEFECTED GROUND STRUCTURE}

In order to show the validity of the equivalent circuit and extracted parameters for the DGS unit, a three-pole LPF with attenuation pole was designed by employing two series DGS unit cells at the cutoff frequency of $3.74 \mathrm{GHz}$ with $0.01 \mathrm{~dB}$ ripple level. Figure 3 shows the equivalent circuit and schematic of the designed LPF with DGS. The parallel capacitance $C_{\mathrm{b}}, 2.67 \mathrm{pF}$, can be realized by using a parallel open stub. The open stub is realized with a T-junction structure where the stub width $w_{1}$ and length $l$ are 6 and $12 \mathrm{~mm}$, respectively. The filter characteristics were simulated and are shown in Figure 4, together with measurement. Good agreements between the simulation and measurement validate the proposed design methodology of the microwave LPF with DGS. In addition, a high-impedance inductance microstrip can be realized by using a microstrip line with a conductor width corresponding to a $50-\Omega$ microstrip with DGS, which can improve the power handling capability of low-pass filters. 

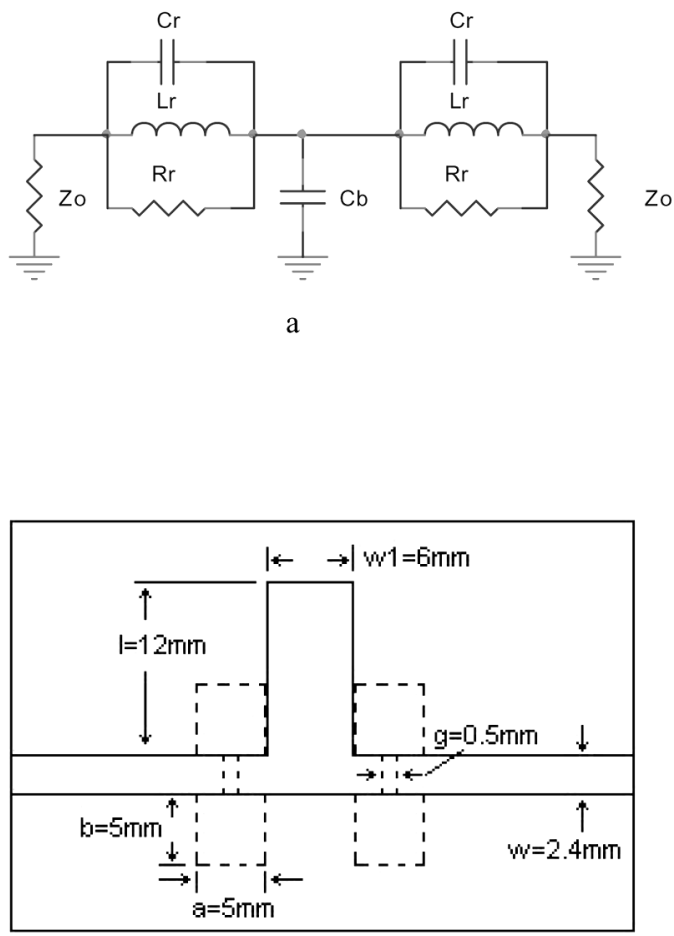

b

FIGURE 3 Equivalent circuit and schematic of the designed LPF with DGS: (a) equivalent circuit of the designed LPF; (b) schematic of the designed LPF.

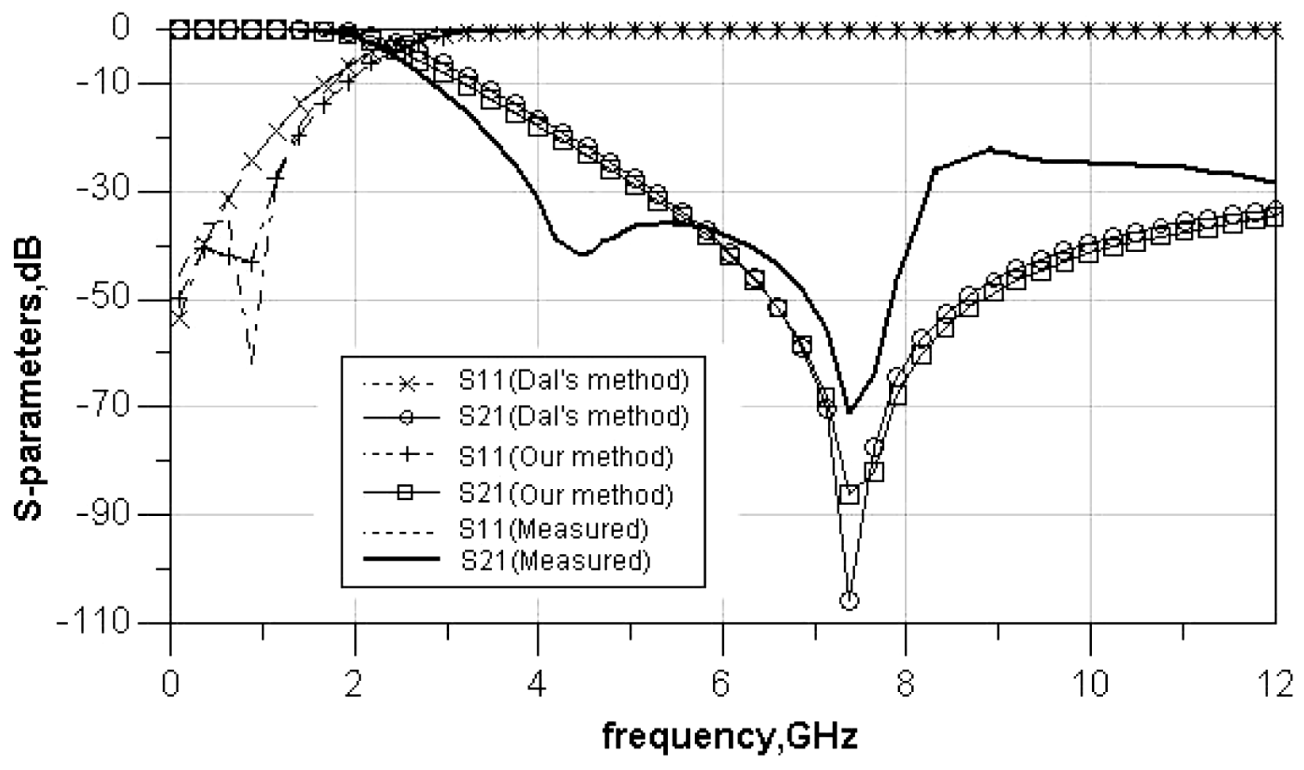

FIGURE 4 Characteristics of the designed LPF with DGS. 


\section{CONCLUSION}

A new and simple parameter-extraction method for the equivalent circuit of DGS is introduced. The systematic analysis and parameter-extraction procedure are described. Further, a microwave LPF with DGS, based on the proposed parameter-extraction method, is designed and implemented. Simulation and measurement of the filter characteristic confirm the validity of the designed LPF. Additionally, simple structure and high power-handling capability are obtained from the proposed LPF.

\section{References}

[1] Lim, J. S., Kim, C. S., Lee, Y. T., Ahn, D. and Nam, S. (2002). Design of lowpass filters using defected ground structure and compensated microstrip line. Electronics Letters, 38, 1357-1358.

[2] Dal, A., Park, J. S., Kim, C. S., Kim, J., Qian, Y. X. and Itoh, T. (2001). A design of the low-pass filter using the novel microstrip defected ground structure. IEEE Trans. MTT, 49, 86-93.

[3] Lim, J. S., Kim, C. S., Lee, Y. T., Dal, A. and Nam, S. (2002). Vertically periodic defected ground structure for planar transmission lines. Electronics Lett., 38, 803-804.

[4] Laso, M. G. (2000). Novel wide and photonic bandgap structure. Microwave and Optical Tech. Lett., 24, 357-360.

[5] Liu, H. W., Li, Z. F., Sun, X. W. and Mao, J. F. (2003). A novel photonic band-gap microstrip structure for low-pass filters of wide stop-band. Microwave and Optical Tech. Lett., 27 (accepted)

[6] Radisic, V., Qian, Y. X., Coccioli, R. and Itoh, T. (1998). Novel 2-D photonic bandgap structure for microstrip lines. IEEE Microwave and Guided Wave Letters, 8, 69-71.

[7] Lim, J. S., Kim, H. S., Ahn, D. and Nam, S. (2001). A power amplifier with efficiency improved using defected ground structure. IEEE Microwave and Guided Wave Letters, 11, 170-172.

[8] Lim, J. S., Park, J. S., Lee, Y. T., Ahn, D. and Nam, S. (2002). Application of defected ground structure in reducing the size of amplifiers. IEEE Microwave and Guided Wave Letters, 12, 261-263.

[9] Lee, Y. T., Lim, J. S., Park, J. S., Ahn, D. and Nam, S. (2002). A novel phase noise reduction technique in oscillators using defected ground structure. IEEE Microwave and Guided Wave Letters, 12, 39-41. 

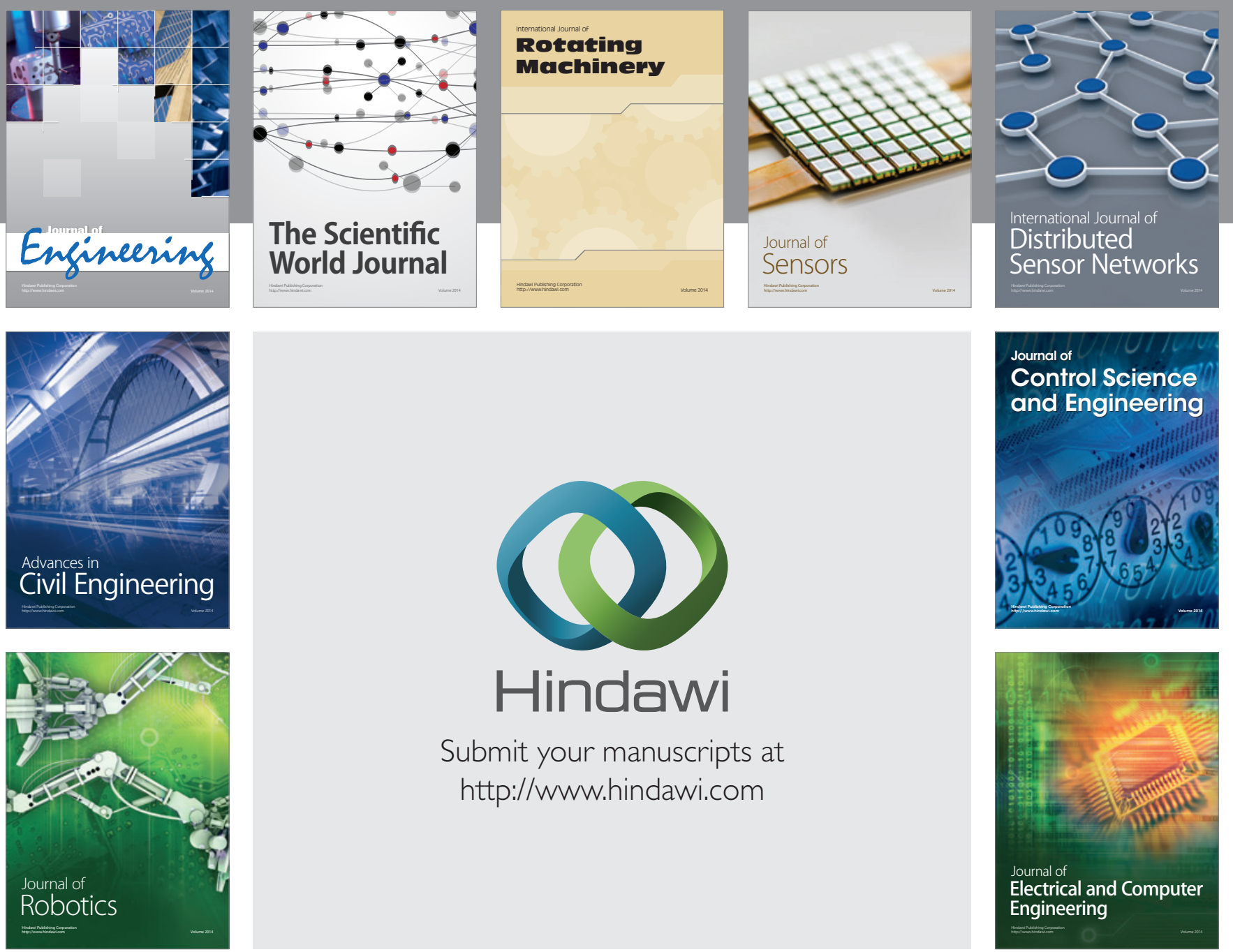

Submit your manuscripts at

http://www.hindawi.com
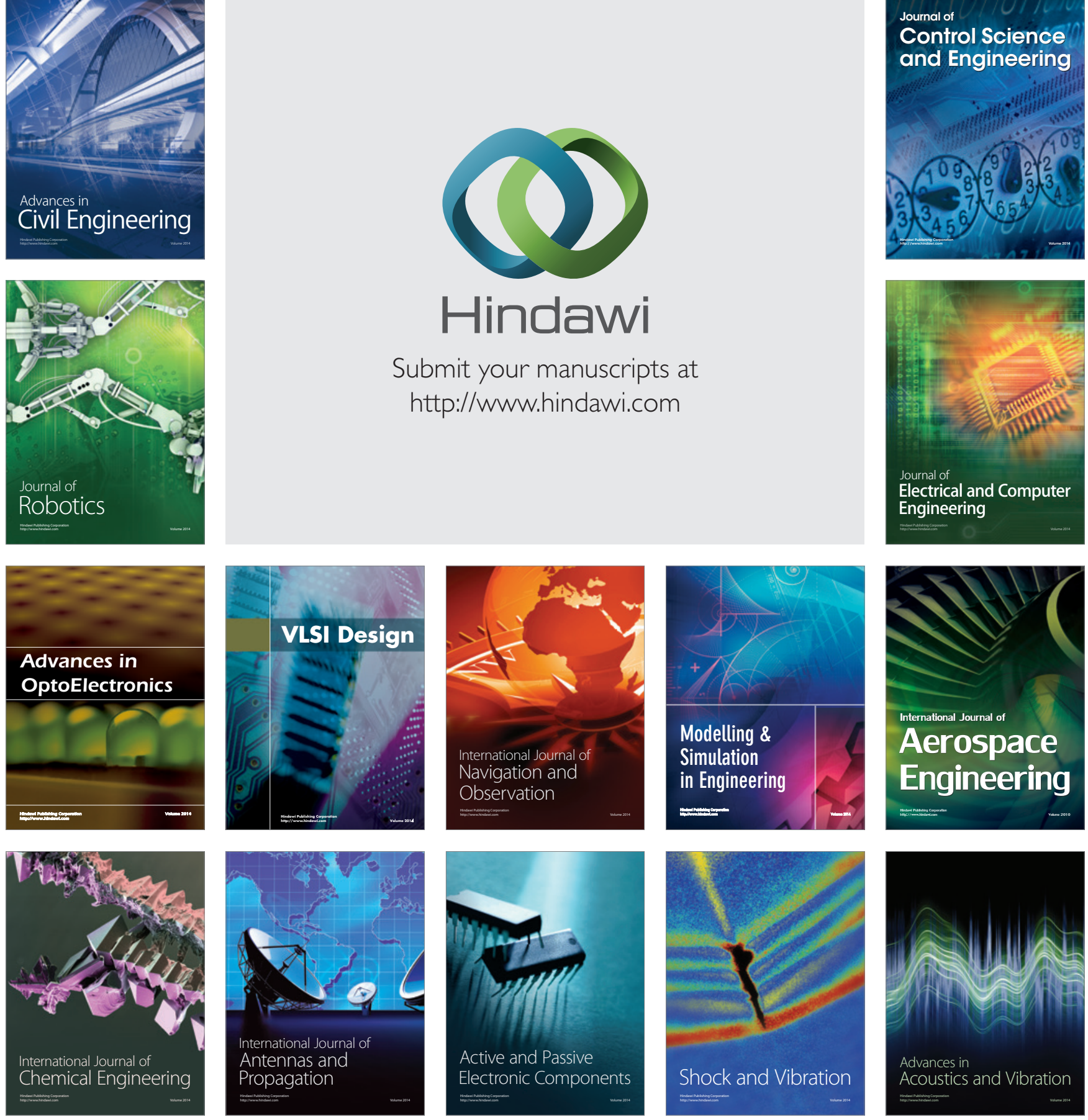